\title{
Predicting terrain contours using a feed-forward neural network
}

\author{
Stephen Erwin-Wright ${ }^{\mathrm{a}}$, David Sanders ${ }^{\mathrm{b}, *}$, Sheng Chen ${ }^{\mathrm{c}}$ \\ ${ }^{a}$ Motiontouch Ltd., Weybridge, Surrey KT13 8LD, UK \\ ${ }^{\mathrm{b}}$ Department of Mechanical and Management Engineering, University of Portsmouth, Anglesea Building, Anglessa Road, Portsmouth, \\ Hampshire PO1 3DI, UK \\ ${ }^{\mathrm{c}}$ Department of Electronics and Computer Science, University of Southampton, Southampton SO17 1BJ, UK
}

\begin{abstract}
Wheeled or tracked vehicles cannot move easily over much of the land surface of the earth. This paper describes research work to create walking machines that are able to travel when the terrain makes wheeled or tracked vehicles ineffective. These legged walking vehicles must be able to negotiate unknown environments with little or no knowledge of the terrain. A predictive terrain contour mapping strategy is proposed that uses a feed-forward neural network trained using a back-propagation algorithm to predict contours based on leg positions and orientations. The strategy is tested using the abilities of a tele-operated eight-legged robot named "Robug $I V$ '. Predicted performance is an improvement on previous implementations and a summarised comparison of the results for the four terrains is provided.
\end{abstract}

(C) 2003 Elsevier Ltd. All rights reserved.

\section{Introduction}

Legged robots can be better than traditional wheeled or tracked vehicles in rough and unstructured terrain (Chen et al., 1999). They can provide greater mobility and better isolation from the irregularities of the terrain, they can be faster and use less fuel and do less environmental damage. Conventional wheeled or tracked vehicles cannot access much of the land surface of the earth (approximately 50\%).

Robug IV (White et al., 1999) is an example of a legged robot. It is a compact, powerful, general-purpose teleoperated eight-legged robot that can walk (ShimMin Song and Waldron, 1988), transition between surfaces, traverse of a $0.305 \mathrm{~m}$-deep pit, and perform autonomous omni-directional climbing. Robug $I V$ is made of aluminium to minimise weight and can carry $50 \mathrm{~m}$ of umbilical with a $5 \mathrm{~kg}$ payload. Humans can easily carry Robug $I V$ as it only weighs $40 \mathrm{~kg}$.

The electromagnetic design concept of the vehicle followed the same design rules and principles that were applied to previous legged robots created during the research, for example Robug III. The vehicles are 'spider like' with a central body and eight peripheral two-link legs. Each leg on Robug $I V$ is $0.7 \mathrm{~m}$ long and consists of

*Corresponding author. Tel.: +44-2392-842565.

E-mail address: david.sanders@port.ac.uk (D. Sanders). two links with four actuated points at the abductor, hip, knee and ankle. The body is 0.3 , long by $0.45 \mathrm{~m}$ wide and $0.4 \mathrm{~m}$ high, and is shown in Fig. 1 .

Orientation of joints in the robot leg are controlled using double-acting pneumatic cylinders. Pneumatic actuators were used because they are lighter and environmentally more rugged than geared electric motors. Force control could be provided by the use of pneumatic actuation. Several design structures were considered, and a kinematic chain like that of the PUMA 560 robot was selected, comprising a vertical first axis of rotation and two mutually parallel horizontal axes for the second and third joints.

Pneumatic cylinders are non-linear devices. Their behaviour varied with the operating temperature. Leaks are common in pneumatic systems and they are difficult to prevent completely. This caused problems during the testing of the abilities of the robot.

Loading on different joints in the robot legs varied widely, for example due to the load being carried by the robot during a walking gait sequence or simply due to the position of a leg.

\section{Prediction of unknown terrain}

The legs of the robot needed to be lifted at the end of an effective stroke, returned, and placed to begin a 


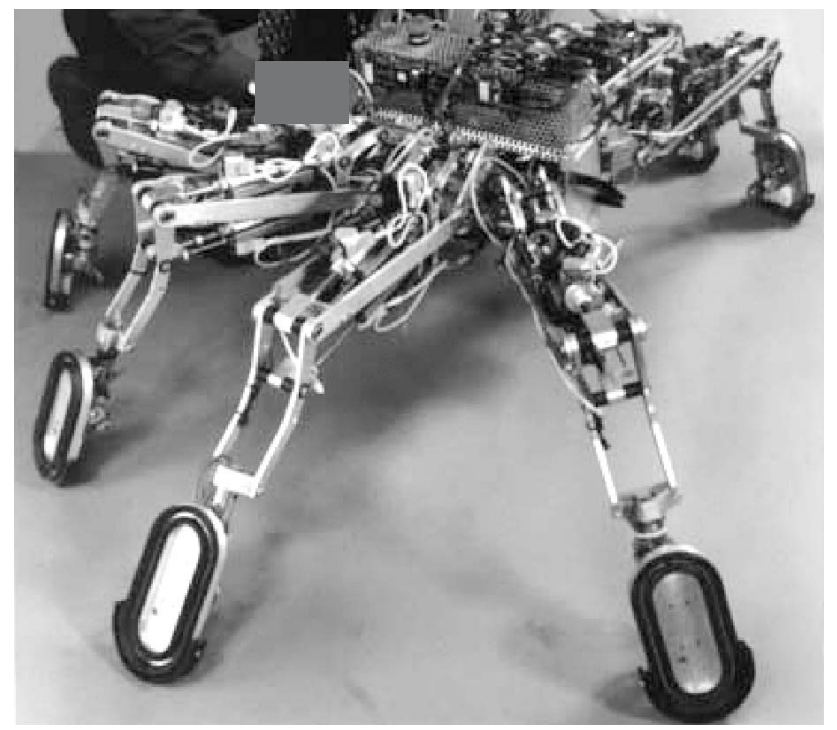

Fig. 1. Robug IV.

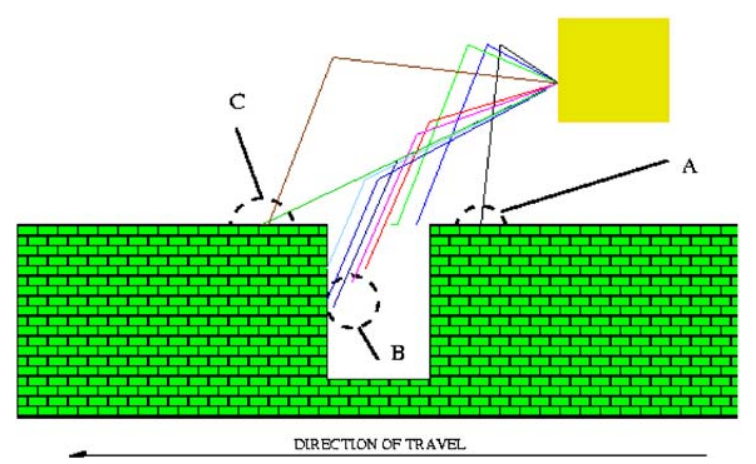

Fig. 2. Moving a foot from A to $\mathrm{C}$.

support stroke. This created a phasing problem defined by the term 'gait'. A Fuzzy-Logic Adaptive Gait (FLAG) algorithm (Galt and Luk, 1997; Lehtokangas, 1999; Kendall et al., 1993) determined when to lift and when to place a leg. The FLAG algorithm was capable of navigating Robug $I V$ across terrains but problems arose because the FLAG algorithm faithfully followed the contours of obstacles. For example, when Robug IV detected a collision during a placement phase, a blind foothold search strategy was executed.

In the situation shown in Fig. 2, the FLAG algorithm deduced that the leg was in the vicinity of the lower kinematic workspace. The course of action to take was to lift the leg higher in the $z$-axis. To execute this process, a small force was exerted in the direction of the detected obstacle during lifting the foot in order to feel for the top surface of the obstacle.

As can be seen in Fig. 2, after rejecting placement B, Robug IV moved the leg and foot a number of times before it placed its foot at C. A terrain mapping system
Table 1

Average prediction error for different types of terrain

\begin{tabular}{lc}
\hline Type of terrain & Average error (in $\mathrm{mm}$ ) \\
\hline Flat surface & 14.2 \\
Linear ramp & 8.8 \\
Steps & 31.2 \\
Random (rough) terrain & 24.1 \\
\hline
\end{tabular}

that could predict unknown terrain could improve such a move by preventing a 'juddery' action during the move from A to C. The system could assist the smooth and efficient walking motions of legged robots in unstructed environments. This is could be important when accuracy of vision-based systems may be reduced in poorly lit or smoke filled environments. In the local area, a predication system may provide more accurate information than a vision system. The better the predictive strategy, the smoother Robug $I V$ 's path, free of turnings and vibrations.

Robug IV already had a terrain mapping system capable of predicting the $z$-axis of the terrain (Warwick et al., 1995). The network used for this was trained for 5000 epochs to predict the next step, based on data from 5 previous steps. The average prediction errors for four real terrain surfaces are shown in Table 1.

The sensors used in the robot had an error after calibration of $\pm 6 \mathrm{~mm}$ so the artificial neural network (ANN) needed to perform within this error. The prediction errors shown in Table 1 were large compared to the sensor error.

The $y$-axis was not predicted.

\section{Improving the strategy using ANNs}

Neural networks are useful for control and prediction problems (Firschein and Fischler, 1987; Sanders et al., 1994, 1996). Their ability to capture and model information from non-linear systems and generalise information from learned data makes them suitable for terrain prediction.

Under certain conditions it may be possible to extend the suitability theories that exist in traditional control theory to systems that include neural networks. This is important if an intelligent control structure for a walking machine is going to be adopted. The proposed new solution used a feed-forward neural network (FFNN), trained using a back-propagation (Jones, 1995) supervised training algorithm. Variables predicted for each leg are shown in Fig. 4 of Urwin-Wright et al. (2002). The layout of Robug $I V$ is shown here in Fig. 3.

If Robug IV were traversing forward in a straight line from right to left as shown in Fig. 2, then it could be assumed that the odd legs (1,3,5 and 7) would encounter 
the same terrains as their opposite legs $(0,2,4$ and 6$)$ within $0.45-1.85 \mathrm{~m}$ (noting that the leg length is 0.7 $(\times 2)$ and width is 0.45$)$.

The new neural network would benefit from having some of these inputs fed into it. Therefore, prediction might only be required for the leading legs.

The FLAG algorithm for Robug $I V$ stated that at least three legs must support the robot before a leg could be lifted and placed. When there were fewer than two legs on either side, Robug $I V$ went into a lockdown state to prevent damage. Assuming the robot was moving then the number of legs on each side of the body could vary from two to four. If the network was designed such that all four legs provided inputs to it but two of these legs might be redundant then what should these inputs be? An example solution is shown in Fig. 4.

Each leg on Robug IV had four Siemens C167 microcontrollers. These micro-processors limited the memory available, so the design had to be compact compared to the power of the desktop PC used for simulation. This constraint needed to be considered when designing the network.

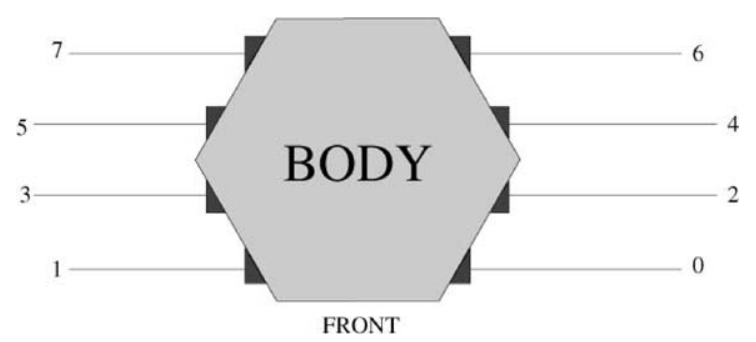

Fig. 3. Plan view of Robug $I V$.

\section{Testing the new strategy}

A simulation called RobSim existed that consisted of an environment with a robot walking in it. The simulation had been used to test the FLAG algorithm that was already incorporated into Robug $I V$. This code had been proved to emulate Robug (Urwin-Wright et al., 2002) so it was decided to use the virtual environment and the models to simulate the operation of Robug $I V$. Past experience had shown that simulation could sometimes suggest that robots and systems can work when they later prove to be unable to cope in practice (Brooks, 1991). Although simulation can sometimes confuse the issue during engineering design, simulation was selected for the initial trials and tests because Robug $I V$ was an expensive piece of equipment, capable of damaging itself. Simulation provided a cost effective, safe and east way to test different gait strategies and especially to reject ideas and algorithms that did not work.

There was also a lack of random workspace to test all the adaptive gait strategies as Robug $I V$ needed to walk for considerable distances over a variety of terrains. The only real way to test Robug $I V$ 's step-climbing ability was take a set of steps and let it traverse them. Problems arose with permission to conduct tests and with moving equipment, air supplies and Robug $I V$ to test sites. The initial work was completed with simulations and a virtual Robug $I V$ and then selected terrains were tested with Robug IV to confirm results.

Initial simulations were conducted without acceleration, which simplified things. The environment consisted of a surface that the robot walked over. The robot needed to be able to find out if a given point in space was above or below the surface, for example whether it

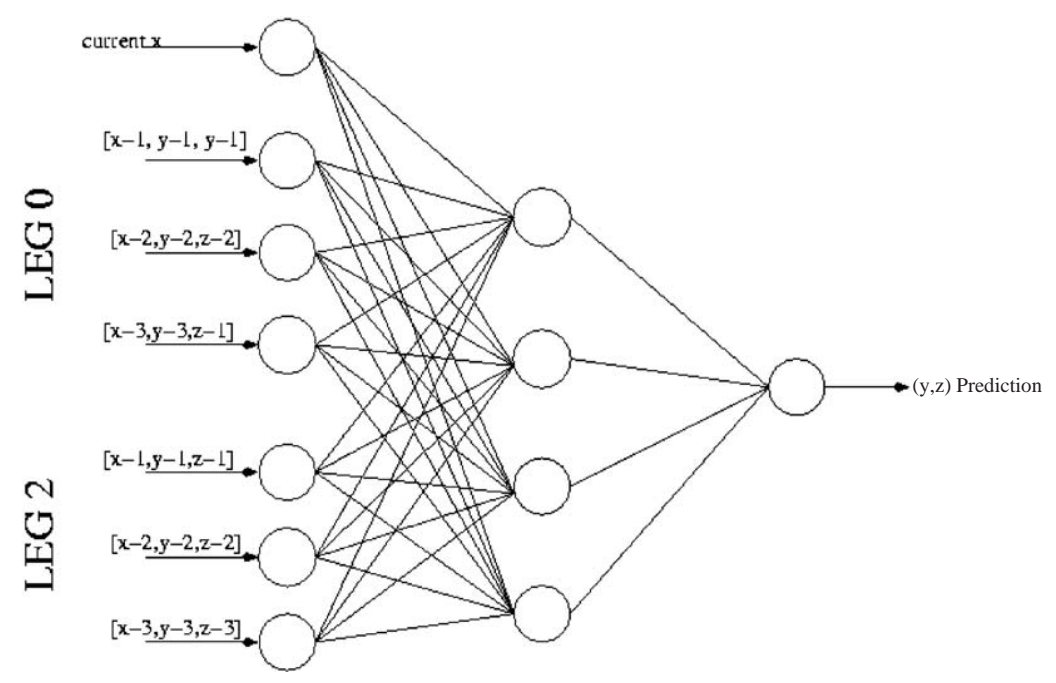

Fig. 4. ANN arrangement to provide a prediction in $y$ and $z$. 
had made contact. The simplest way to model the various surfaces was as a series of planar tiles.

Training and testing were similar as the training sequence was close to the testing sequence and both came from the same source. Using RobSim, data was acquired for a range of terrains. As RobSim had been proved, the mathematical calculations remained the same. The $x$-step that was originally used was $0.26 \mathrm{~m}$ (Galt and Luk, 1997); therefore one step was roughly $0.26 \mathrm{~m}$. This data was then formulated into previous step, next step, training and testing data.

Testing routes were $4000 \mathrm{~m}$ long to provide sets of samples, that was roughly 15,000 steps. From this, the training and testing were derived. These sets were then applied to a genetic FFNN (Goldberg, 1988; Luk et al., 2000). The number of inputs was then varied along with the numbers of hidden neurons in order to find the optimum configuration; within the constraints mentioned earlier in Section 3. Rules developed for one leg in Fig. 3 were applied to the other legs. As an example, the results for leg 0 are shown in the next section but the rules for leg zero were applied to the other seven; leg 0 was used for initial testing and then results were implemented on the other legs.

It was not possible for Robug $I V$ to manoeuvre with fewer than two legs per side. Robug $I V$ went into a lockdown state when this occurred. To overcome the problem of which legs to feed into the networks, the network was designed with only two legs for the inputs. So the previous steps from leg 2 constituted the other input to the network.

\section{Results}

Testing took place over the routes to provide the sets of samples. These sets were then applied to the genetic FFNN. The number of inputs was varied along with the numbers of hidden neurons in order to find the optimum configuration. The optimum number of input and hidden layers neurons was defined as the point when no more advantage was identified in increasing their number during the testing phase. The first value to be set was the number of input neurons. Galt and Luk (1997) and Urwin-Wright et al. (2002) stated that the number of hidden layers should be four, trained for 5000 epochs. The number of previous steps that were being fed back into the network ranged from one to three. Above three, the size of the FFNN became unworkable within the constraints of the robot systems; below one, the FFNN became inane. The RMS prediction of the $y$ - and $x$-axis plotted against the number of hidden neurons is shown in Figs. 5 and 6.

From the RMS prediction error of the $y$-axis shown in Fig. 5, all the previous steps fed into the network appear in ascending order. Using more than two hidden

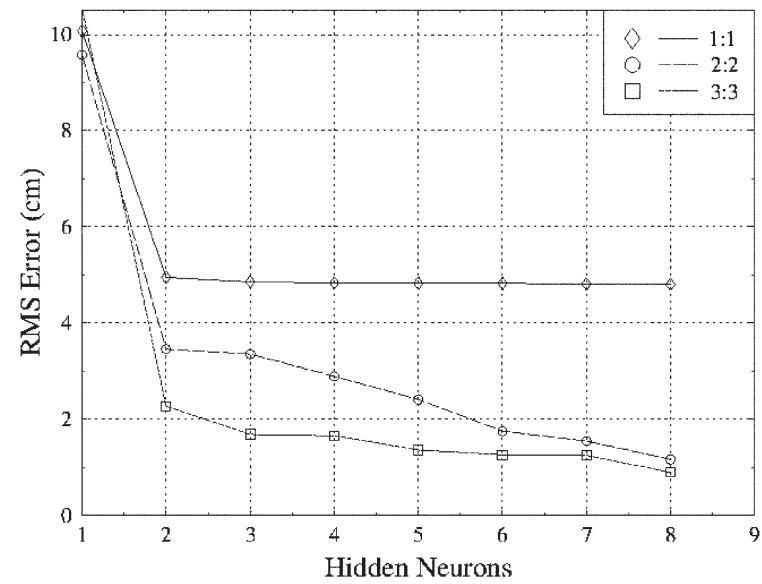

Fig. 5. $y$-Axis RMS error over all terrains.

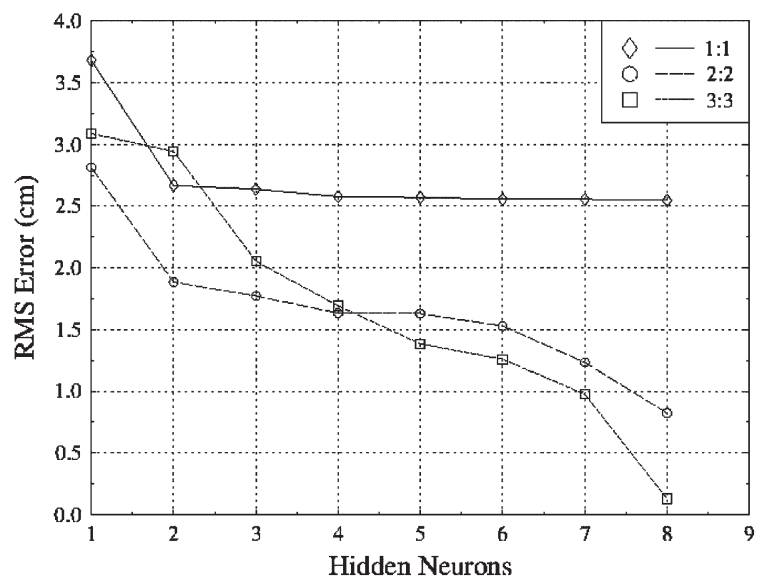

Fig. 6. $z$-Axis RMS error over all terrains.

neurons significantly reduced the error of the prediction, and using more than eight reduced the RMS of the prediction to less than $1.0 \mathrm{~cm}$.

The RMS prediction of the $z$-axis shown in Fig. 6 is interesting. Feeding three previous steps into the network and having less than four hidden neurons resulted in the network performing worse than when two previous steps are fed into the network. As with only using two previous steps, having more than eight hidden neurons reduced the RMS error to below $1.0 \mathrm{~cm}$ and even close to $0.25 \mathrm{~cm}$.

Considering the error of the pneumatic cylinder once it had been calibrated, which was $\pm 6 \mathrm{~mm}$; to reduce the RMS error to within this amount would require at least eight hidden neurons and three previous steps to be fed into the network. Problems arose here, as achieving this result increased the demand on the micro-controllers. It was decided than an RMS error of $1.6 \mathrm{~cm}$ was tolerable; taking advantage of the fact that when using two previous steps the network performance was better. So two previous steps were fed were into the FFNN, with four hidden neurons. The following results show now 
the network performed over the four terrains listed in Table 1.

The figures illustrating these results use error in $\mathrm{cm}$ rather than error squared. Because the error is small, squaring made the graphics appear as straight lines in the paper.

\subsection{Predicting the $y$-axis}

Fig. 7 shows the position of the leg in the $y$-axis when Robug $I V$ was walking up a ramp. The error in the prediction of this ramp is shown in $\mathrm{cm}$ in Fig. 8; the RMS error of this is $0.450374 \mathrm{~cm}$.

Fig. 9 shows the position of the leg in the $y$-axis when Robug IV was walking across rough terrain. The error in the prediction of this terrain is shown in $\mathrm{cm}$ in Fig. 10; the RMS error of this is $2.418234 \mathrm{~cm}$.

Fig. 11 shows the position of the leg in the $y$-axis when Robug $I V$ was walking across smooth terrain. The error in the prediction of this terrain is shown in $\mathrm{cm}$ in Fig. 12; the RMS error of this is $0.422875 \mathrm{~cm}$.

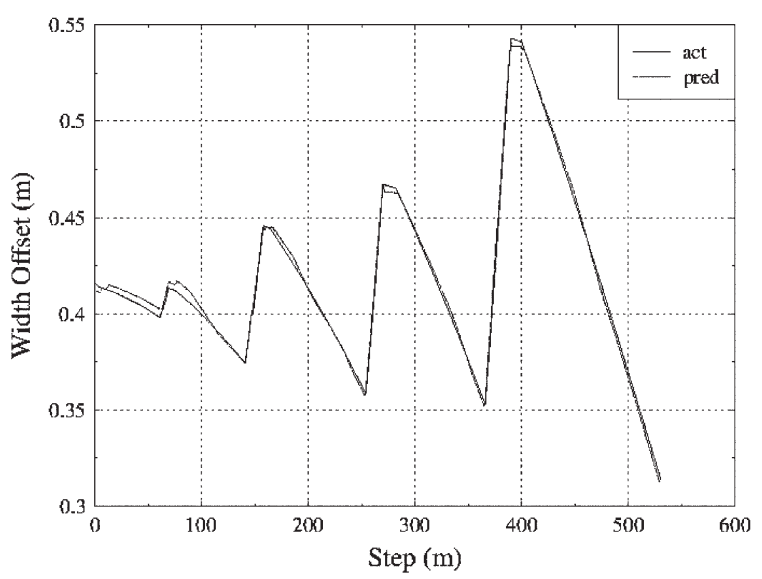

Fig. 7. Ramp terrain.

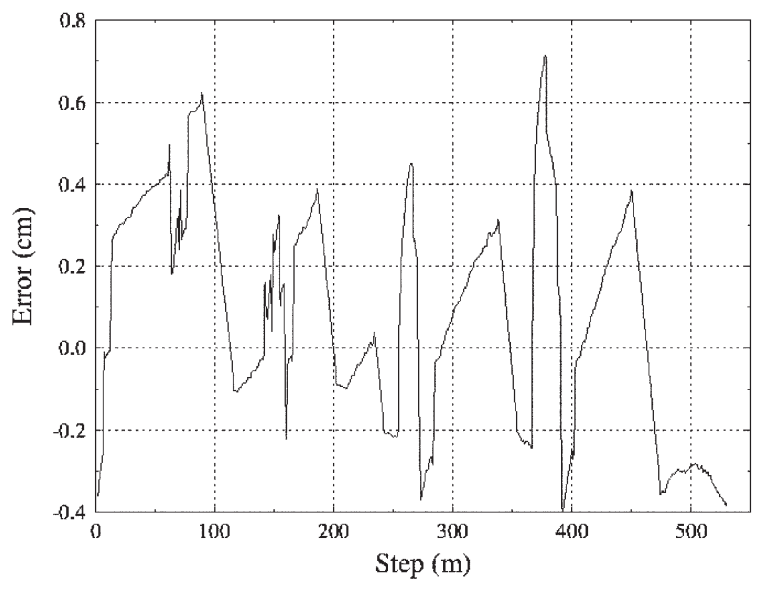

Fig. 8. Position error for $y$-axis over the ramp terrain.

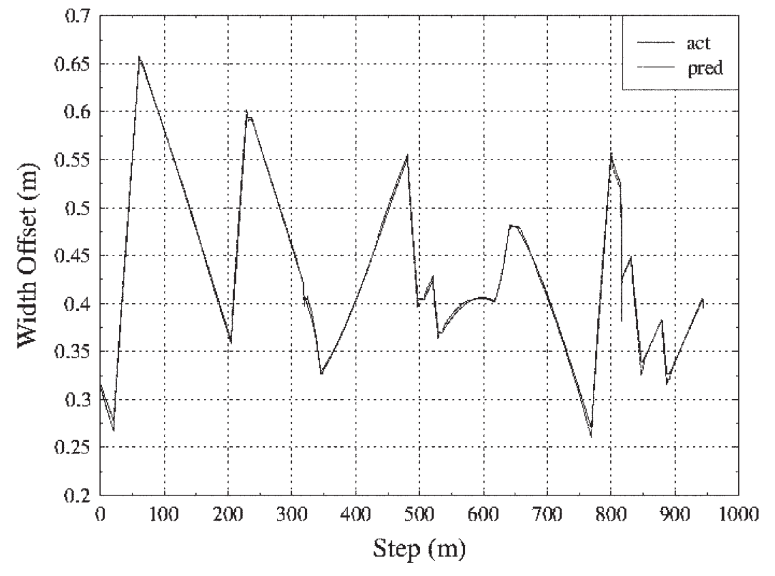

Fig. 9. Rough terrain.

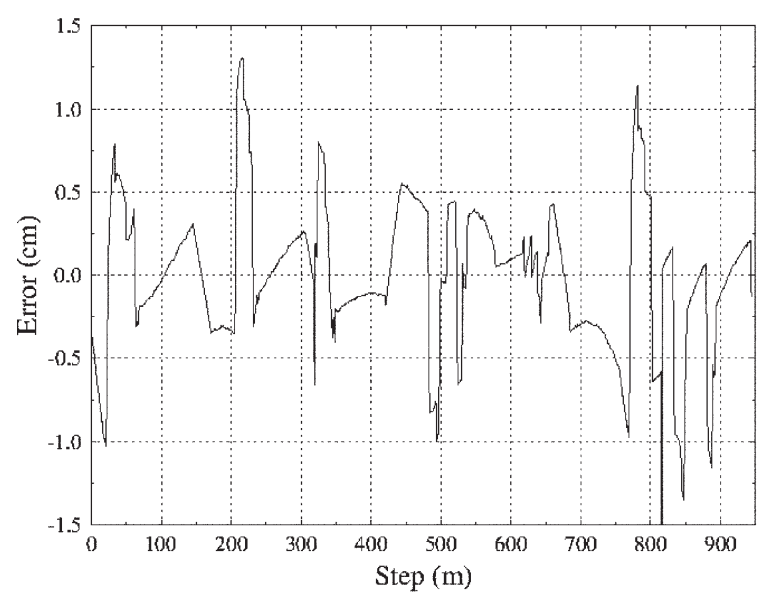

Fig. 10. Position error for $y$-axis over the rough terrain.

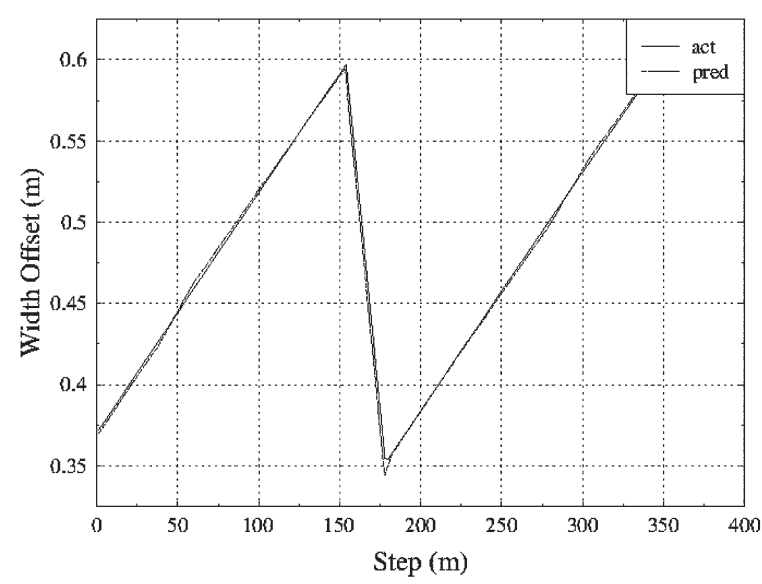

Fig. 11. Smooth terrain.

Fig. 13 shows the position of the leg in the $y$-axis when Robug $I V$ was walking up a set of stairs is shown in $\mathrm{cm}$ in Fig. 14; the RMS error of this is $8.256384 \mathrm{~cm}$. 


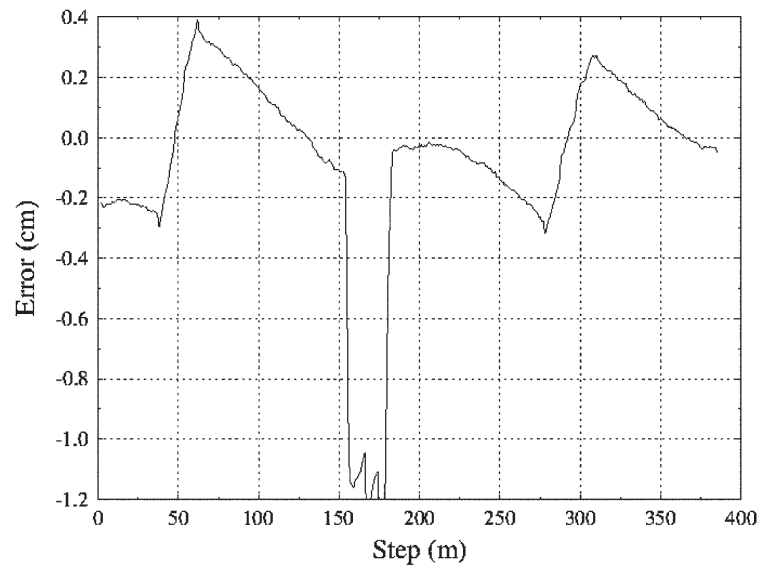

Fig. 12. Position error for $y$-axis over the smooth terrain.

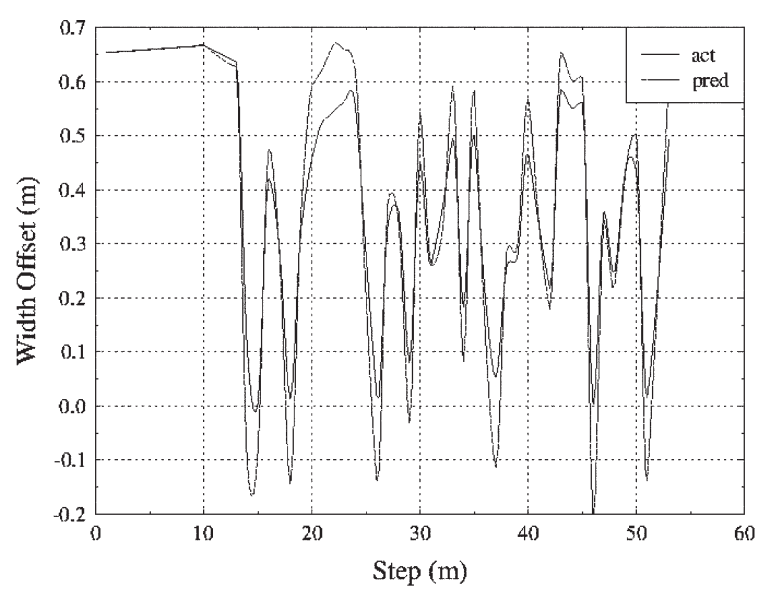

Fig. 13. Stair terrain.

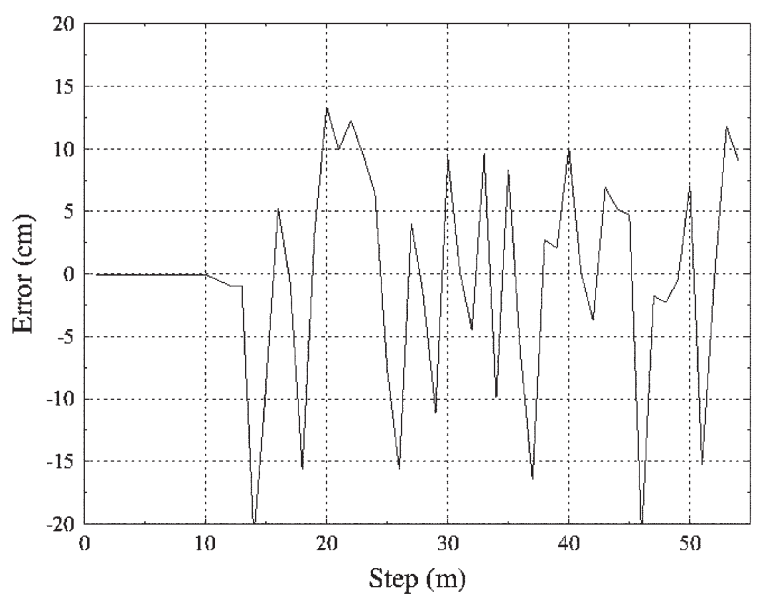

Fig. 14. Position error for $y$-axis over the stair terrain.

\subsection{Predicting the z-axis}

Fig. 15 shows the position of the leg in the $z$-axis when Robug $I V$ was walking up a ramp. The error in the

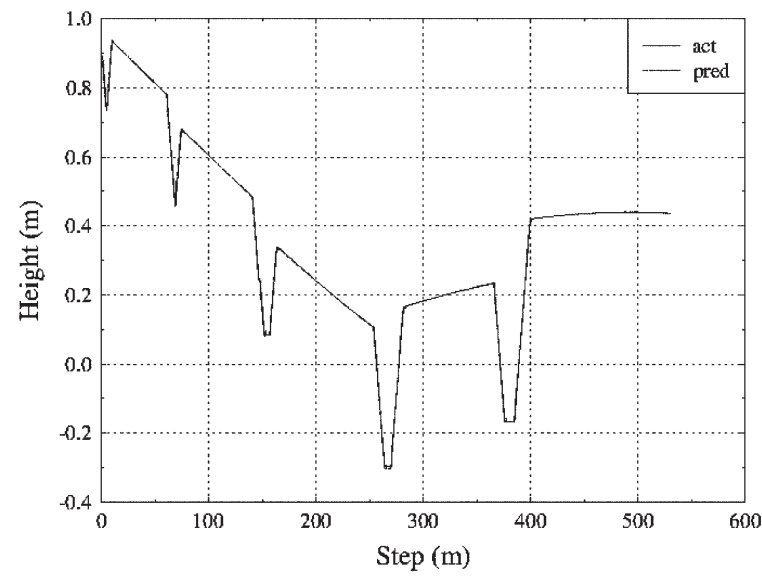

Fig. 15. Ramp terrain.

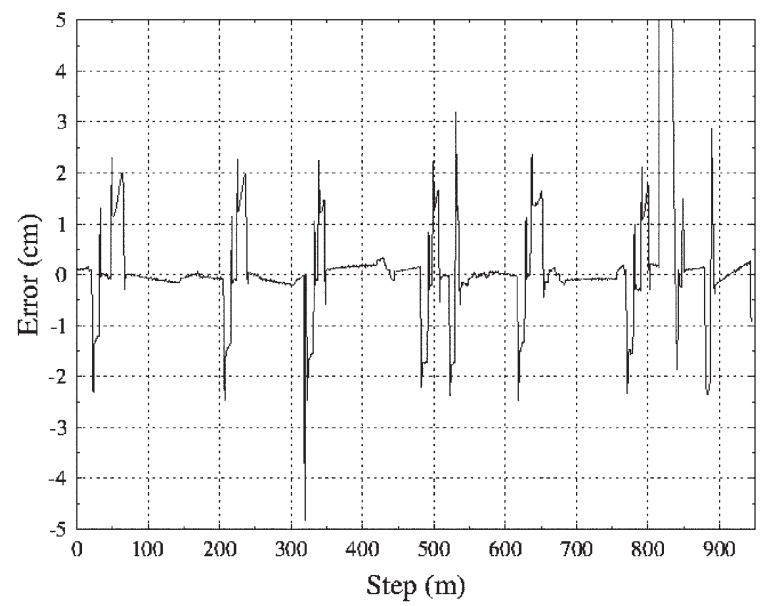

Fig. 16. Position error for $z$-axis over the ramp terrain.

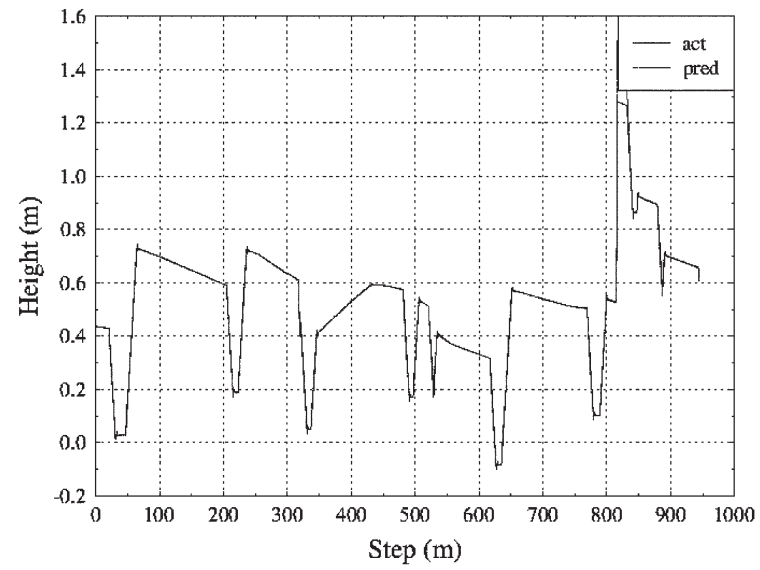

Fig. 17. Rough terrain.

prediction of this ramp is shown in $\mathrm{cm}$ in Fig. 16; the RMS error of this is $2.541913 \mathrm{~cm}$.

Fig. 17 shows the position of the leg in the $z$-axis when Robug $I V$ was walking across rough terrain. The error in 


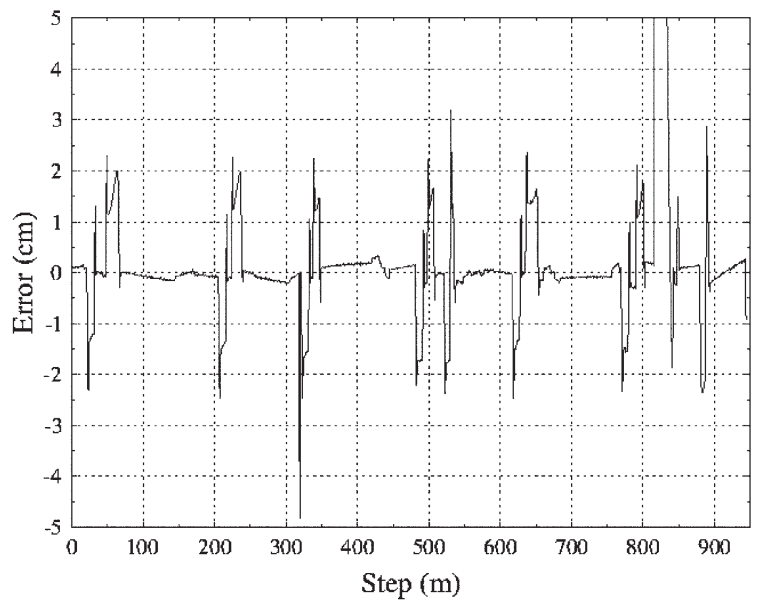

Fig. 18. Position error for $z$-axis over the rough terrain.

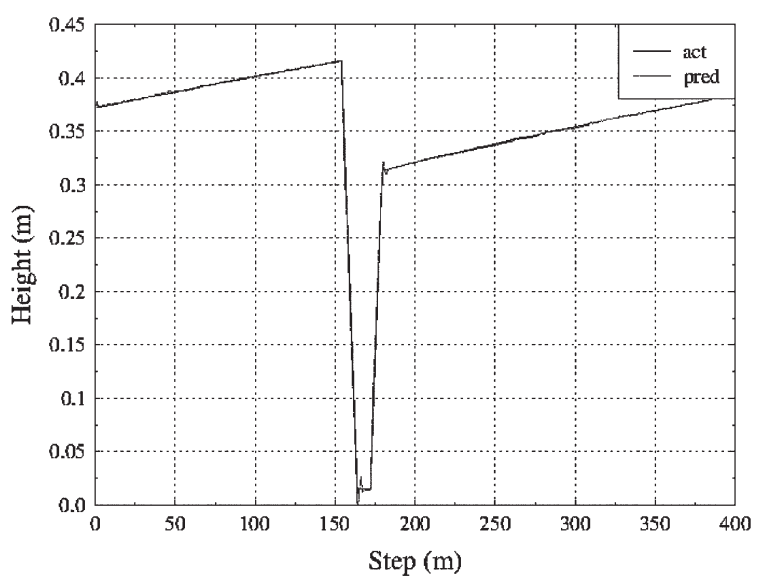

Fig. 19. Smooth terrain.

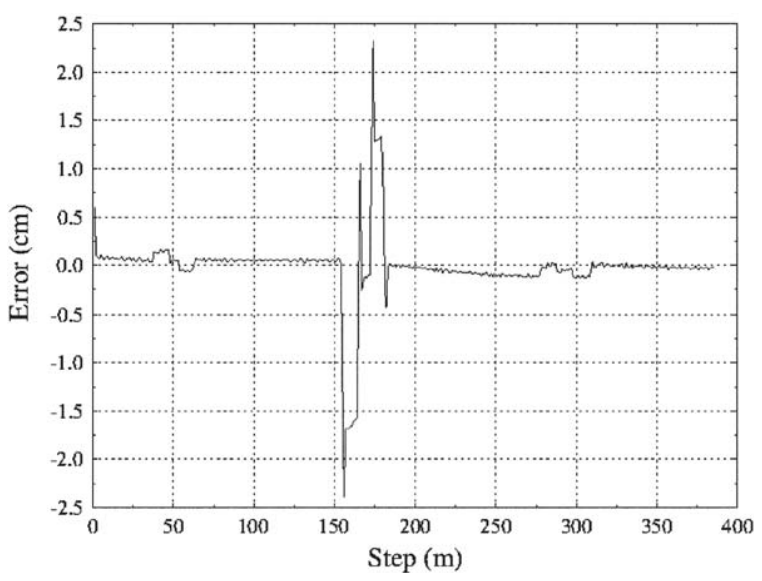

Fig. 20. Position error for $z$-axis over the ramp terrain.

the prediction of this terrain is shown in $\mathrm{cm}$ in Fig. 18 . The RMS error of this is $2.541913 \mathrm{~cm}$.

Fig. 19 shows the position of the leg in the $z$-axis when Robug $I V$ was walking across smooth terrain. The error

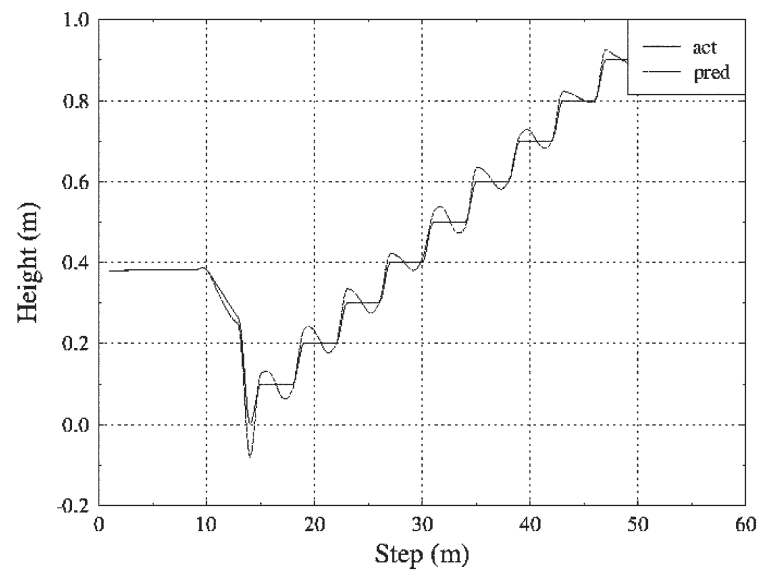

Fig. 21. Stair terrain.

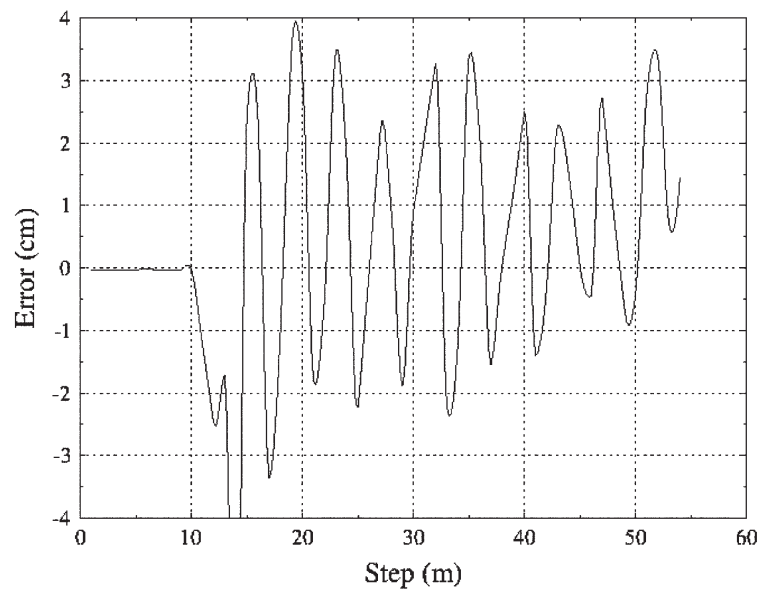

Fig. 22. Position error for $z$-axis over the stair terrain.

in the prediction of this terrain is shown in $\mathrm{cm}$ in Fig. 20; the RMS error of this is $0.352966 \mathrm{~cm}$.

Fig. 21 shows the position of the leg in the $z$-axis when Robug IV was walking up a flight of stairs. The error in the prediction of these stairs is shown in $\mathrm{cm}$ in Fig. 22; the RMS error of this is $2.541913 \mathrm{~cm}$.

\subsubsection{Learning rate (epochs)}

The two best predictions were obtained with four and eight hidden neurons. The learning rate of the three different inputs is shown in Figs. 23 and 24.

Fig. 23 suggests that there is no significant advantage in network learning with more than 2000 epochs, as the RMS prediction error does not improve much.

With eight hidden neurons the network learning levels off at around 2600 epochs as shown in Fig. 24. As only four hidden neurons were used, the optimum epoch training sequence had only 2000 epochs. 


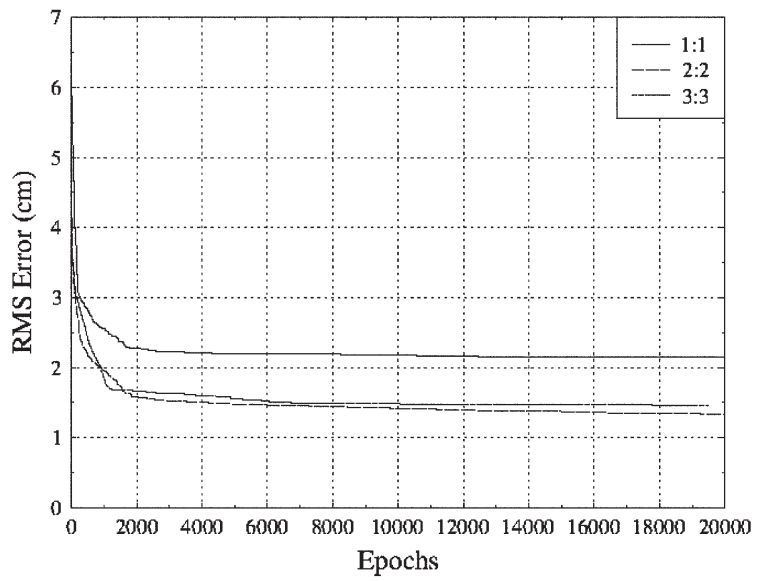

Fig. 23. Network learning with four hidden neurons.

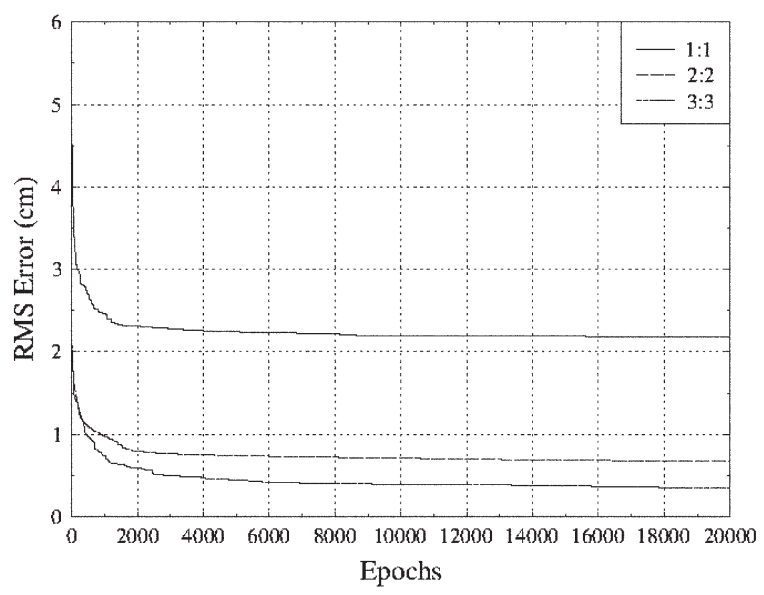

Fig. 24. Network learning with eight hidden neurons.

\section{Conclusion}

The terrain prediction did not predict the $y$-axis location of the robot legs, so there is no comparison for the $y$-axis prediction; a summary of results is given in Table 2.

A summary of the results for the $z$-axis prediction comparisons for four terrains is shown in Table 3.

Assuming that the terrains tested in the old implementation are similar to those tested with new FFNN, then the new network performed better than the old network on rough, smooth and stair surfaces. The results for the ramp terrain shown in Fig. 15 are not completely linear, and this may account for the poorer result in the $z$-axis. It was also assumed that 'average error' quoted in the previous result was actually the RMS error. The number of epochs is significantly smaller. The old implementation used 5000 epochs; the new implementation uses less than half that, at 2000 epochs. This reduced implementation time.
Table 2

$y$-Axis error for different types of terrain

\begin{tabular}{ll}
\hline Type of terrain & RMS prediction error in $\mathrm{cm}$ \\
\hline Stair & 8.2564 \\
Smooth & 0.4229 \\
Rough & 2.4182 \\
Ramp & 0.4504 \\
Collective & 2.887 \\
\hline
\end{tabular}

Table 3

Comparisons of $z$-axis error for different types of terrain

\begin{tabular}{lll}
\hline $\begin{array}{l}\text { Type of } \\
\text { terrain }\end{array}$ & $\begin{array}{l}\text { RMS error of new } \\
\text { prediction in } \mathrm{cm}\end{array}$ & $\begin{array}{l}\text { Average error of } \\
\text { old prediction in } \mathrm{cm}\end{array}$ \\
\hline Stair & 2.1348 & 3.12 \\
Smooth & 0.353 & 1.42 \\
Rough & 1.4984 & 2.41 \\
Ramp & 2.5418 & 0.88 \\
Collective & 1.6321 & 1.96 \\
\hline
\end{tabular}

\section{References}

Brooks, R., 1991. Intelligence without reason. MIT AI Lab memo 1(1293) http://www.leglab.ai.nit.edu.

Chen, S., Istepanian, R., Gait, S., Luk, B., 1999. Intelligent Walking Gait Generation for Legged Robots. Professional Engineering Publishing, Bury St. Edmunds, UK.

Firschein, O., Fischler, M., 1987. The Eye, The Brain and The Computer. Addison-Wesley, UK and USA.

Galt, S., Luk, B., 1997. Predictive terrain contour mapping for a legged robot. Fifth International Conference on Artificial Neural Networks, University of Reading, UK, pp. 129-133.

Goldberg, D.E., 1988. Genetic Algorithms in Search, Optimisation and Machine Learning. Addison-Wesley, Reading, MA.

Jones, A., 1995. Neural networks and genetic algorithms for prediction and control of dynamic systems. Technical Report, Imperial College, London.

Kendall, W.S., Barndorff-Nielsonm, O.E., Jensen, J.L., 1993 Networks and Chaos-Statistical and Probabilistic Aspects. Chapman \& Hall, London.

Lehtokangas, T.M., 1999. Additive neural networks and periodic patterns. Neural Networks 12 (4-5), 617-626.

Luk, B.L., Gait, S., Chen, S., 2001. Using genetic algorithms to establish efficient walking gaits for an eight-legged robot. International Journal of Systems Science 32 (6), 703-713.

Sanders, D.A., Haynes, B.P., Vogt, M., Stott, I.J., 1994. Control of a robot using neural networks as feed forward estimators and as feedback controllers. Proceedings of European Robotics and Intelligent Systems Conference, Vol. (B), Spain, pp. 1065-1074.

Sanders, D.A., Haynes, B.P., Tewkesbury, G.E., Stott, I.J., 1996. The addition of neural networks to the inner feedback path in order to improve on the use of pre-trained feed forward estimators. Journal of Mathematics and Computers in Simulation 41, 461-472.

Song, S.-M., Waldron, K., 1988. Machines that Walk: the adaptive suspension vehicle. MIT Press, Cambridge, MA.

Urwin-Wright, S., Chen, A., Sanders, D.A., 2002. Terrain prediction for an eight-legged robot. Journal of Robotic Systems 19 (2), 91-98.

Warwick, K., Irwin, G., Hunt, K., 1995. Neural Network Applications in Control, IEEE Control Engineering Series, Vol. 53. Academic Press, Exeter, England.

White, T., Luk, B., Cooke, D.S., Hewer, N., 1999. Implementation of Modularity in Robug IV-Preliminary Results. Professional Engineering Publishing, Bury St. Edmunds, UK. 